\title{
Potenciais efeitos das mudanças climáticas futuras sobre a distribuição de um anuro da Caatinga Rhinella granulosa (Anura, Bufonidae)
}

\author{
Hauanny Rodrigues Oliveira ${ }^{1} \&$ Fernanda A. S. Cassemiro ${ }^{2}$
}

1. Universidade Federal de Goiás, ICB1, Departamento de Ecologia, 74001-970, Goiânia, Goiás, Brasil. (hauannyoliveira@gmail.com)

2. Systema Naturae Consultoria Ambiental, rua 58, Jardim Goiás, 74810-250, Goiânia, Goiás, Brasil. (fernandacassemiro@gmail.com)

\begin{abstract}
RESUMO. Neste estudo, usamos dois tipos de modelagem de distribuição de espécies (correlativo e mecanístico), com o objetivo de avaliar o efeito das mudanças climáticas sob a distribuição geográfica de Rhinella granulosa (Spix, 1824), espécie inserida principalmente no bioma Caatinga. Avaliamos a predição, levantada por outros autores, de que espécies de anfíbios distribuídos em climas quentes terão suas distribuições espaciais restringidas por aumento da temperatura considerando cenários futuros. Na abordagem correlativa, os resultados mostraram que as distribuições espaciais geradas pelo modelo de distância Euclidiana foram mais conservativas, ou seja, as áreas que apresentaram menor distância do nicho ótimo se restringiram às áreas de distribuição real da espécie (Caatinga) e às pequenas regiões que abrangem o bioma Cerrado. A abordagem mecanística apresentou resultados menos conservativos, onde o habitat indicado como adequado para $R$. granulosa está contido em grande parte da América do Sul, formando uma extensa área contínua. No geral, verificou-se que $R$. granulosa não sofrerá forte influência climática sobre sua distribuição geográfica no futuro, pelo menos até 2080, provavelmente por apresentar uma fisiologia extremamente tolerante às altas temperaturas e por possuir adaptações para suportar clima quente e seco.
\end{abstract}

PALAVRAS-CHAVE. Modelagem de distribuição de espécies, tolerância termal, modelo correlativo, modelo mecanístico.

ABSTRACT. Potential effects of climate change on the distribution of a Caatinga's frog Rhinella granulosa (Anura, Bufonidae). In this study, we used two types of species distribution modelling (correlative and mechanistic) in order to evaluate the effects of climate change on the geographic distribution of Rhinella granulosa (Spix, 1824), distributed in the Caatinga biome. We tested the prediction that amphibians distributed in warm weather will have their spatial distribution constrained by high temperatures in the future. Using the correlation approach, we observed that the potential distribution generated by Euclidian Distance showed more conservative areas (e.g. with a smaller distance from optimum niche) limiting it to the current distribution of the species (e.g. Caatinga), and to small areas in the Cerrado biome. The mechanistic approach showed a less conservative result, in which the habitat indicated as suitable for $R$. granulosa comprised a large extension of South America, encompassing a contiguous area. In general, we observed that the spatial distribution of $R$. granulosa would not be strongly affected by climate change, at least until 2080. Probably, this species has a tolerant physiology to high temperatures and shows adaptations that support dry and hot weather.

KEYWORDS. Species distribution modelling, thermal tolerance, correlative model, mechanistic model.

As alterações climáticas têm contribuído para mudanças acentuadas na distribuição geográfica das espécies ao longo das últimas décadas (WALTHER et al., 2002; Root et al., 2003; Parmesan, 2006); consequentemente, a distribuição das espécies tem sido foco de estudos importantes na área da Ecologia, principalmente nos últimos 20 anos (Deutsch et al., 2008; Diniz-FilHo et al., 2010; BuCKLEY et al., 2010).

A área de distribuição é o resultado da soma das posições dos indivíduos no espaço, sendo assim, um atributo emergente de uma espécie. Portanto, a área de distribuição de uma espécie é a convergência de diversos aspectos da biologia e ecologia, incluindo tamanho corporal, densidade populacional, potencial de dispersão, disponibilidade de recursos, interações ecológicas, restrições fisiológicas e mecanismos adaptativos que moldam a espécie ao longo de sua história evolutiva (Brown et al., 1996). Compreender a importância de cada um desses fatores e o grau de interação entre eles na estruturação espacial das distribuições das espécies é de interesse primordial para assegurar a efetiva conservação da biodiversidade.

De acordo com WhitTaKer et al. (2001), fatores climáticos exercem papel fundamental sobre a distribuição das espécies em grandes escalas geográficas. A confirmação da assertiva acima veio com o advento de novas técnicas computacionais e estatísticas, que se baseiam em características ambientais de pontos de ocorrência de uma espécie para estimar a sua distribuição potencial, podendo ser usado como indicativo da sua tolerância ambiental e preferências de hábitat (Deutsch et al., 2008; Kearney et al., 2010). Desta forma, os modelos de distribuição de espécies têm sido amplamente usados para predizer áreas adequadas para o estabelecimento de espécies sob projeções climáticas presente e futura, objetivando identificar regiões nas quais ações preventivas devam ser tomadas (BEAUMONT et al., 2008; FrankLIN, 2010; Nori et al., 2011).

Especificamente, os modelos de distribuição de espécies combinam pontos de ocorrência de indivíduos dentro de sua área conhecida com dados climáticos oriundos da mesma área para estimar a amplitude de variação ambiental que é adequado para a espécie (GuISAN \& Thuiller, 2005; Franklin, 2010; HARTLEY et al., 2010). Assim, é possível estimar a atual distribuição de uma espécie e predizer áreas que possuem características ambientais iguais ou semelhantes. Em geral, esse tipo de modelo é elaborado utilizando o mecanismo de nicho grineliano (SOBERón, 2007), o qual delimita o conjunto de condições ambientais adequadas para uma determinada espécie, formando um envelope bioclimático. Este é construído de acordo com valores máximos e mínimos para todas as variáveis ambientais usadas, buscando valores que se repetem 
e, assim, mostrando a adequabilidade da espécie para um determinado local. A correlação entre os pontos de ocorrência da espécie e as variáveis ambientais, define o que denominamos de modelo correlativo (BUCKLEY et al., 2010). Contudo, esse tipo de modelo tem sido criticado principalmente em relação a sua simplicidade de correlacionar somente os dados ambientais aos de distribuição das espécies (BUCKLEY et al., 2010), ignorando processos fisiológicos, por exemplo.

$\mathrm{Na}$ tentativa de melhorar o poder preditivo dos modelos de distribuição potencial das espécies, os modelos mecanísticos têm sido cada vez mais utilizados (Chown \& Gaston, 2008; Kearney \& Porter, 2009; BuCKLey et al., 2010; Franklin, 2010). Esse tipo de modelo define a distribuição potencial de uma dada espécie baseando-se nos limites de tolerância fisiológica, ou seja, processos dominantes que regem a sobrevivência e reprodução são considerados para predizer a distribuição geográfica (KEARNER \& PorTER, 2009; BuCKLEY et al., 2010). Sabe-se que dentre vários fatores ambientais que restringem a sobrevivência de uma espécie, a temperatura é um dos mais importantes (COSTA et al., 2012; Ribeiro \& NAVAS, 2012). Diante disso, a tolerância termal vem sendo usada com mais frequência na elaboração de modelos mecanísticos (KEARNEY et al., 2010; BuCKLEY et al., 2010), pois através dos limites mínimo e máximo de temperatura pode-se indicar, por exemplo, quanto determinada espécie suportaria se houvesse um aumento nas temperaturas ambientais sem perder significativamente seu desempenho (Deustch et al., 2008).

Há fortes evidências que determinadas espécies tenham mudado o período de seus ciclos de vida durante o ano, bem como suas distribuições espaciais (Kearney et al., 2008; Beaumont et al., 2009; Thomas, 2010; LOYOLA \& MARTINs, 2011). Isto provavelmente esteja ligado às variações anuais e de longo prazo na temperatura (Sinervo et al., 2010; CHEN et al., 2011). Animais ectotérmicos são altamente suscetíveis às alterações climáticas, como é o caso dos anfíbios (Costa et al., 2012). Extremos de temperatura podem alterar o metabolismo desses animais de forma direta e indireta, afetando processos essenciais aos seus ciclos de vida. Diante desse cenário de mudanças climáticas, estudos que visam compreender os processos que atuam em grande (e.g., clima) e pequena escala (e.g., fisiologia) são de suma importância na elucidação de questões ecológicas, bem como na decisão de medidas de conservação (KEARNEY et al., 2010; BUCKLEY et al., 2010).

De acordo com Costa et al. (2012), estudos fisiológicos indicam que não existe relação direta entre temperatura média da área de ocorrência de uma espécie e seu grau de tolerância a acréscimos de temperatura (NAVAs et al., 2008). Nesse caso, o fator relevante na definição da resposta biológica ao aquecimento, é a relação entre o limite de tolerância térmica de cada espécie e a amplitude do aquecimento esperado para o futuro. Desta forma, anfíbios de habitats quentes e áridos provavelmente já estão vivendo muito próximo do seu limiar térmico máximo (KATZENBERGER et al., 2012). Neste estudo, utilizamos dois diferentes tipos de abordagem de modelagem de distribuição de espécies (correlativa e mecanística), com o objetivo de avaliar o efeito das mudanças climáticas sob a distribuição geográfica de Rhinella granulosa (Spix, 1824), espécie inserida principalmente no bioma Caatinga, onde o clima é quente e árido. Assim, avaliamos a predição levantada por KATZENBERGER et al. (2012), de que espécies de anfíbios distribuídas em climas quentes terão suas distribuições espaciais restringidas por aumento da temperatura considerando cenários futuros.

Anfíbios são animais com grande sensibilidade e considerados bioindicadores da qualidade ambiental; nesse sentido, este estudo se torna importante para averiguar a resposta de $R$. granulosa frente às mudanças climáticas futuras. A modelagem da distribuição potencial de $R$. granulosa baseou-se em diferentes modelos climáticos, e os resultados foram projetados em toda a América do Sul considerando dois períodos de tempo (presente e futuro).

\section{MATERIAL E MÉTODOS}

Dados de distribuição da espécie e ambientais. Rhinella granulosa ocorre ao norte da Região Sudeste, sendo amplamente distribuída na Região Nordeste do Brasil, abrangendo toda a Caatinga e poucos registros dessa espécie foram observados na Mata Atlântica (Navas et al., 2007; Narvaes \& Rodrigues, 2009). De acordo com Leal et al. (2003), a Caatinga ocupa uma área aproximada de $800.000 \mathrm{~km} 2$, sendo que cerca de $50 \%$ desta área recebe menos de $750 \mathrm{~mm}$ de chuva por ano. Outra característica marcante da Caatinga é a temperatura média anual elevada, com valores entre 26 a $28^{\circ} \mathrm{C}$ (Leal et al., 2003). Rodrigues (2003) afirmou que há 49 espécies de anfíbios registradas neste bioma.

Indivíduos de $R$. granulosa podem atingir até $76 \mathrm{~mm}$ de comprimento, e experimentos feitos em laboratório mostraram que adultos conseguiram tolerar altas temperaturas $\left(42,5 \pm 1,8^{\circ} \mathrm{C}\right.$; NAVAS et al., 2007).

Para a elaboração dos modelos de distribuição potencial de $R$. granulosa, 71 pontos de ocorrência (NARVAes \& Rodrigues, 2009) não redundantes foram sobrepostos à uma malha geográfica com 1558 células de $1^{\circ}$ de latitude e longitude sobre a América do Sul.

Cenários climáticos. Três cenários climáticos foram usados para a elaboração dos modelos de distribuição potencial. O primeiro deles é de projeção presente, o qual utilizamos dados de (a) temperatura média anual, temperatura média mensal, (b) temperatura máxima do mês mais quente, (c) temperatura mínima do mês mais frio, (d) temperatura média do trimestre mais chuvoso, (e) temperatura média do trimestre 
mais seco, (f) temperatura média do trimestre mais quente, (g) temperatura média do trimestre mais frio e (h) precipitação anual. Todas essas variáveis foram projetadas sobre a América do Sul e utilizadas como variáveis ambientais preditoras da distribuição da espécie.

Os outros dois cenários foram utilizados para projeções futuras. Especificamente nestes cenários, os dados climáticos para a modelagem de distribuição de espécies foram derivados de três modelos de circulação oceânico-atmosférica (Atmosphere-Ocean General Circulation - AOGCMs): CCCma, CSIRO e HadCM3. Apesar de outros AOGCMs estejam disponíveis, esta seleção abrange diferentes predições e foi definido para maximizar os graus de diferença do aquecimento global predito (veja DinIZ-FilHo et al., 2009). Para cada AOGCMs, projeções climáticas sobre dois cenários de emissão de gases de efeito estufa foram usados, um pessimista (A2) e outro otimista (B2) (TRENBERTH, 2005) projetados para o ano de 2080. Os dados climáticos dos cenários presente e futuro foram obtidos através da base de dados WorldClim (www.worldclim.org).

Mapas que mostram o delta da temperatura, ou seja, a diferença entre a temperatura presente e as temperaturas dos cenários futuros otimista (B2) e pessimista (A2), nos permitem visualizar a região na América do Sul onde haverá maior mudança na temperatura (Fig. 1).

Modelagem de distribuição de espécies. Para a construção dos modelos correlativos aplicamos o método de Distância Euclidiana, utilizando o software BIOENSEMBLES (veja DinIZ-FILHo et al., 2009; RANGEL et al., 2009), o qual consiste em medir a similaridade de cada ponto de ocorrência em relação à média (ou ao centro) do espaço ecológico, gerando, assim, um envelope circular. Desta forma, os valores mais altos apresentados no mapa referem-se a locais mais distantes do nicho ótimo da espécie, ao passo que valores mais baixos representam locais mais adequados à sobrevivência da espécie e semelhantes ao nicho ótimo. Através da abordagem de modelagem correlativa, foram gerados três mapas, um representando a distribuição potencial para o presente e outros dois para o futuro (cenário pessimista e otimista).

Os modelos de distribuição elaborados através da abordagem mecanística basearam-se na tolerância termal de Rhinella granulosa. Esta foi obtida experimentalmente por NaVAs et al. (2007), que definiram as temperaturas críticas máxima e mínima nas quais o desempenho locomotor (velocidade e distância de saltos) de indivíduos adultos e juvenis de $R$. granulosa diminui ou cessa. Os autores supracitados concluíram que para $R$. granulosa o desempenho locomotor decresce substancialmente abaixo de $20^{\circ} \mathrm{C}$ e acima de $42,5^{\circ} \mathrm{C}$. Sendo assim, consideramos esses extremos como a margem de tolerância termal para $R$. granulosa, haja vista que a locomoção é um requisito importante para a perpetuação da espécie (NAVAS et al., 2007).

$\mathrm{Na}$ abordagem mecanística, a presença foi atribuída somente nas células cuja temperatura média

\section{Delta de Temperatura}

\section{Diferença entre cenários atual e futuros}
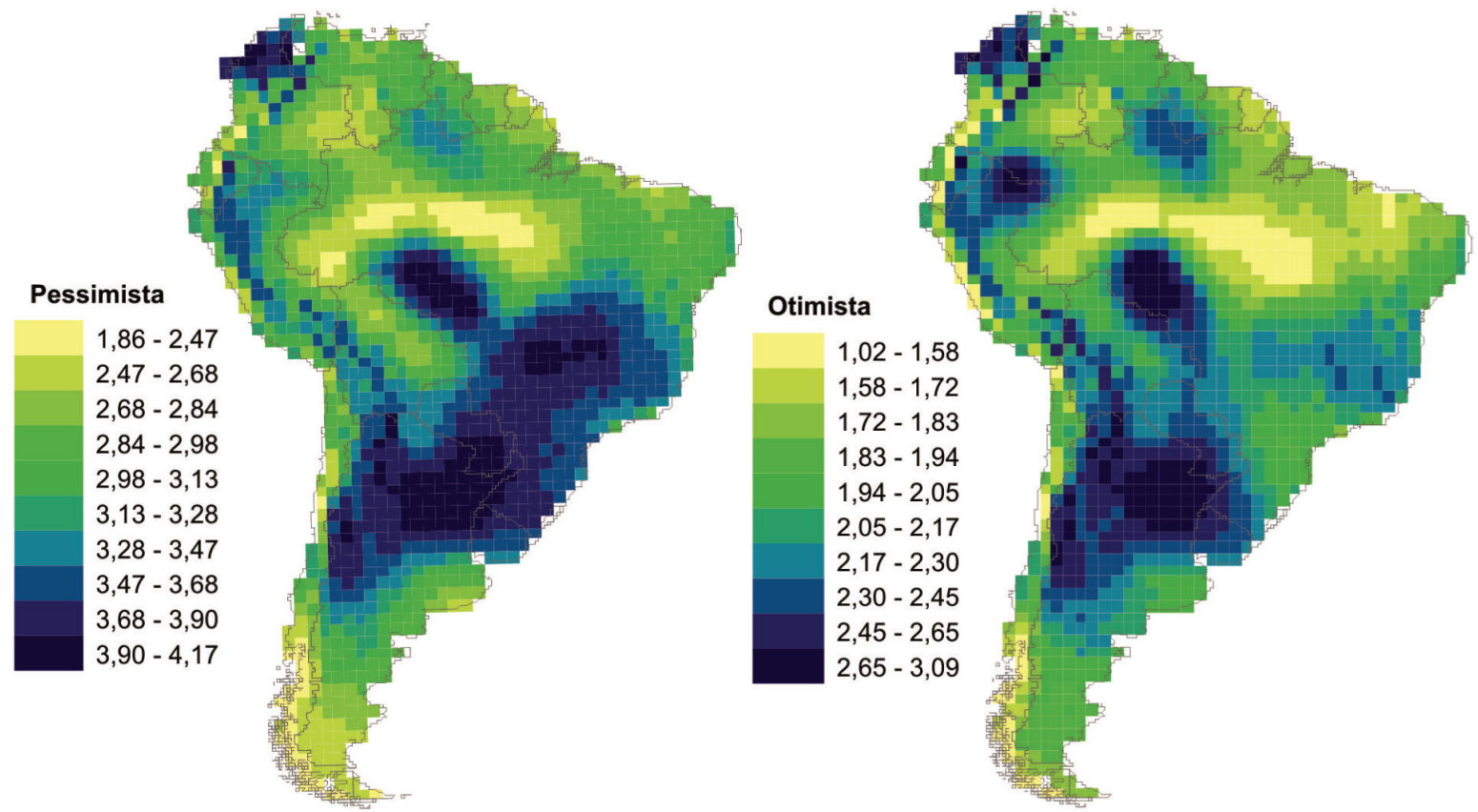

Fig. 1. Mapas de delta de temperatura (diferença entre temperatura presente e temperatura dos cenários futuros ${ }^{\circ} \mathrm{C}$ ) para cenários pessimista e otimista. 
anual, temperatura máxima do mês mais quente e temperatura mínima do mês mais frio sobrepuseram à faixa de temperatura estabelecida como tolerância termal, definindo então o limite biofísico da área de ocorrência da espécie. Desta forma, através dessa abordagem foram gerados três mapas de distribuição potencial, um representando o cenário presente e os outros dois representando cenários futuros (pessimista e otimista).

As duas abordagens de modelagem nos permitem comparar os diferentes modelos de distribuição potencial de $R$. granulosa. Os modelos que utilizam características fisiológicas (tolerância termal) baseiam-se na definição de limites de tolerância em relação às variáveis referentes a várias medidas de temperatura ambiental, permitindo definir um envelope bioclimático. Por outro lado, os modelos que se baseiam no método de Distância Euclidiana permitem gerar um envelope circular ao redor do ótimo no espaço ecológico, permitindo interpretar o modelo como uma expressão das restrições ambientais que a espécie sofre, incluindo as correlações entre variáveis (De Marco JR. \& SiQueIRA, 2009). Portanto, a principal diferença entre as duas abordagens é o fato de que nos modelos mecanísticos os limites máximos e mínimos em relação às variáveis ambientais são definidos experimentalmente e não com base em dados de ocorrência.

Validação e comparação dos modelos. As predições geradas pelos modelos de distribuição potencial de Rhinella granulosa foram comparadas e validadas aplicando-se basicamente os índices de sensitividade e o de especificidade (FrankLIn, 2010; BuCKLEY et al., 2010; CASSEMiro et al., 2012). O índice de sensitividade é mensurado por meio da proporção das presenças reais corretamente preditas (razão entre presenças preditas e o número total de presenças reais; MANEL et al., 2001). O índice de especificidade é a proporção das ausências reais corretamente preditas (razão entre ausências preditas e o número total de ausências reais; MANEL et al., 2001). O desempenho do modelo associa os dois índices acima, calculando a porcentagem de todos os casos que são corretamente preditos (presenças reais mais ausências reais dividido pelo total de casos; MANEL et al., 2001). Além desses índices, através dos mapas de distribuição potencial foi permitido fazer uma comparação visual entre os modelos

\section{RESULTADOS}

Os mapas de distribuição potencial que utilizam a abordagem mecanística (Fig. 2), mostraram que o habitat indicado como adequado para Rhinella granulosa está contido em grande parte da América do Sul - do norte da Argentina ao norte da Venezuela -, formando uma extensa área contínua. Quando a projeção presente é comparada com cenários futuros, observa-se um pequeno aumento na distribuição da espécie, principalmente no cenário pessimista, no qual a distribuição potencial de $R$. granulosa se estende mais ao sul do continente.

Os mapas gerados pelo modelo de Distância Euclidiana (Fig. 3) foram mais conservativos, ou seja, as áreas que apresentaram menor distância do nicho ótimo (regiões em amarelo nos mapas) se restringiram às áreas de distribuição real da espécie (Caatinga) e às pequenas regiões que abrangem o bioma Cerrado (Fig. 3). Quando se compara os três cenários climáticos, observa-se que no cenário pessimista há uma leve retração da distribuição de $R$. granulosa (veja também Fig. 4). É importante

\section{Modelos Mecanísticos}
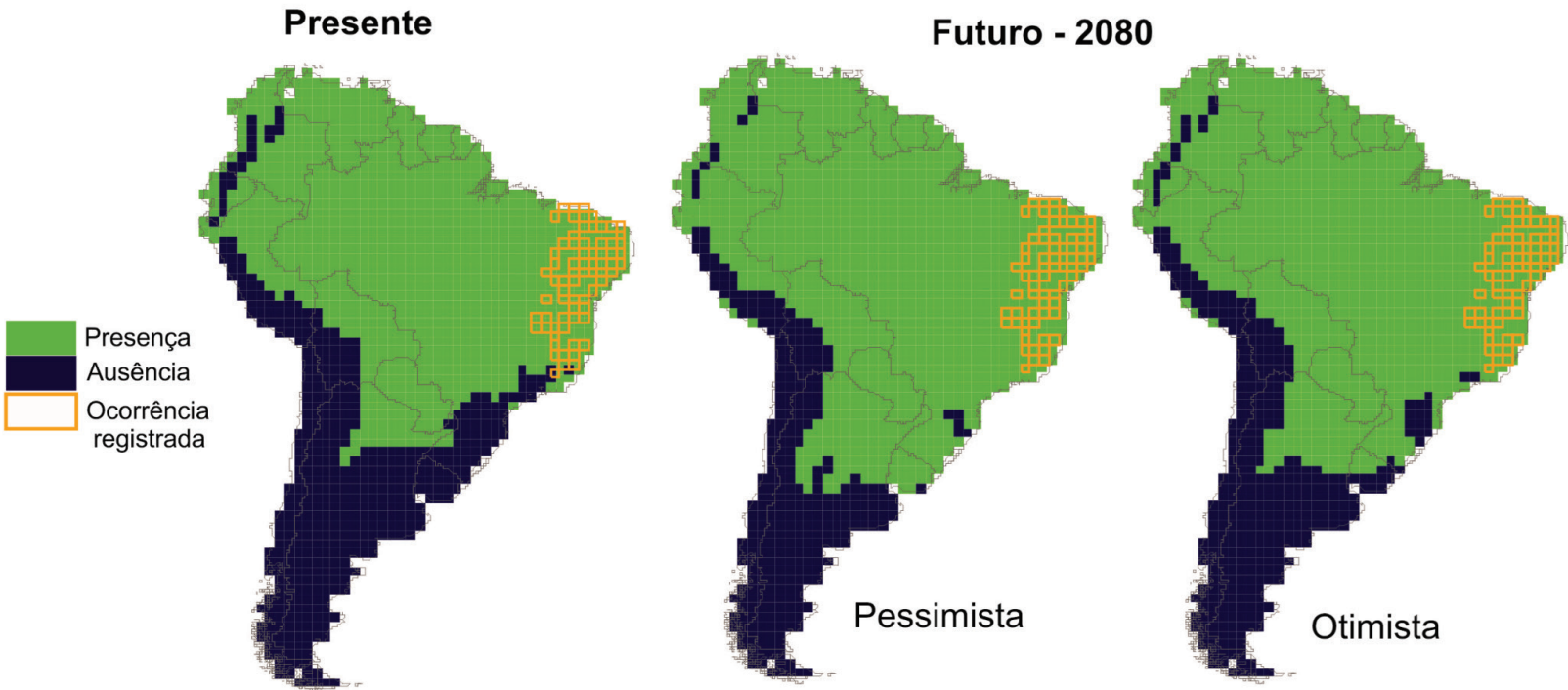

Fig. 2. Mapas das distribuições potenciais de Rhinella granulosa (Spix, 1824) gerados através dos modelos mecanísticos, considerando os diferentes cenários climáticos. 


\section{Modelos correlativos}
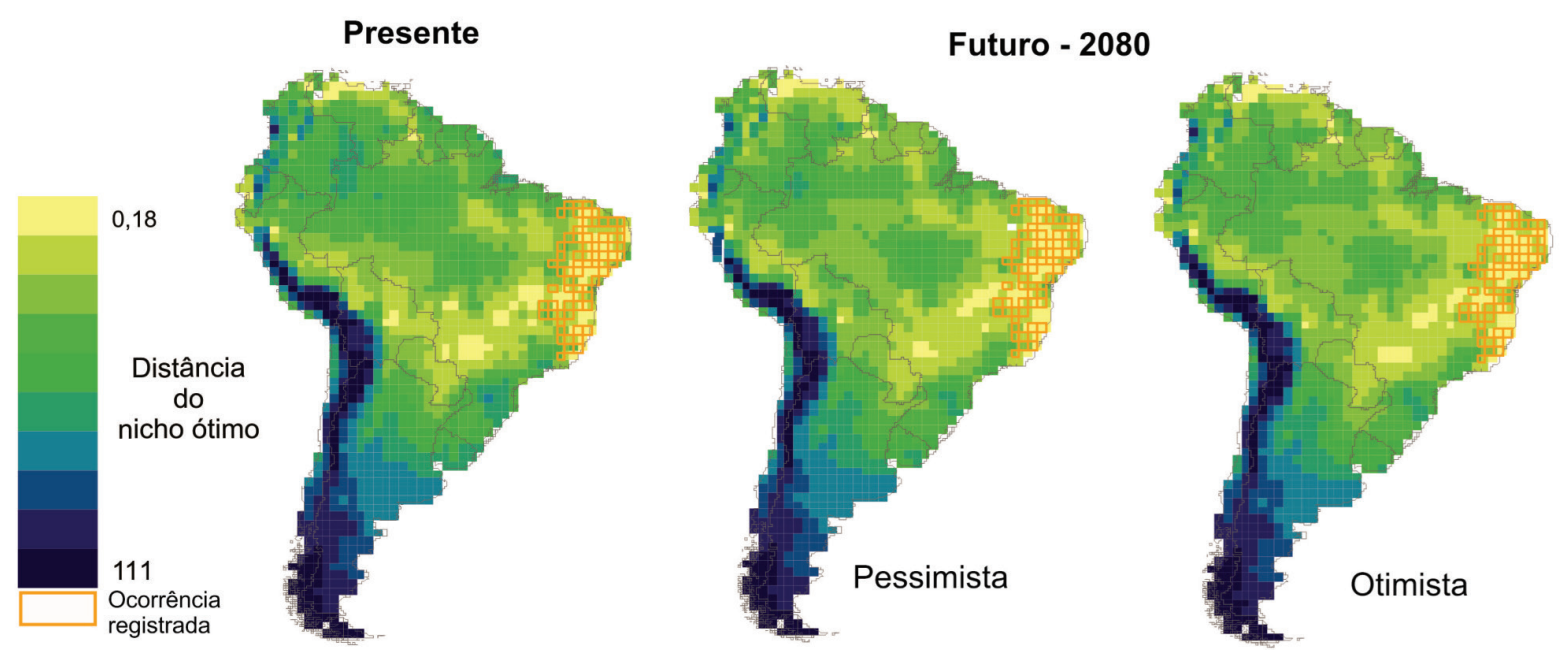

Fig. 3. Mapas das distribuições potenciais de Rhinella granulosa (Spix, 1824) gerados através de modelos correlativos, considerando os cenários pessimista e otimista.

salientar que os três cenários sob a abordagem correlativa apresentaram áreas de maior adequabilidade (áreas em amarelo e verde) que se assemelham às áreas dentro dos limites de tolerância termal da espécie (Fig. 2).

A Figura 4 mostra de forma mais clara a variação da distribuição da espécie, considerando os dois tipos de abordagens de modelagem e os três cenários climáticos. De fato, os modelos mecanísticos são menos conservativos do que os correlativos, estimando a distribuição potencial da espécie em aproximadamente $80 \%$ da América do Sul. Ao contrário, as estimativas de distribuição dos modelos correlativos não ultrapassam a $25 \%$ de toda área estudada. Contudo, para esses modelos, é válido ressaltar que foram considerados somente valores de distância euclidiana $\leq$ 15 como sendo áreas mais adequadas para a distribuição potencial de $R$. granulosa (Fig. 3 para ver a área com distância $\leq 15$ ).

Os índices comparativos permitem uma melhor aferição entre os modelos (Tab. I). Entre os modelos correlativos houve grande variação no índice

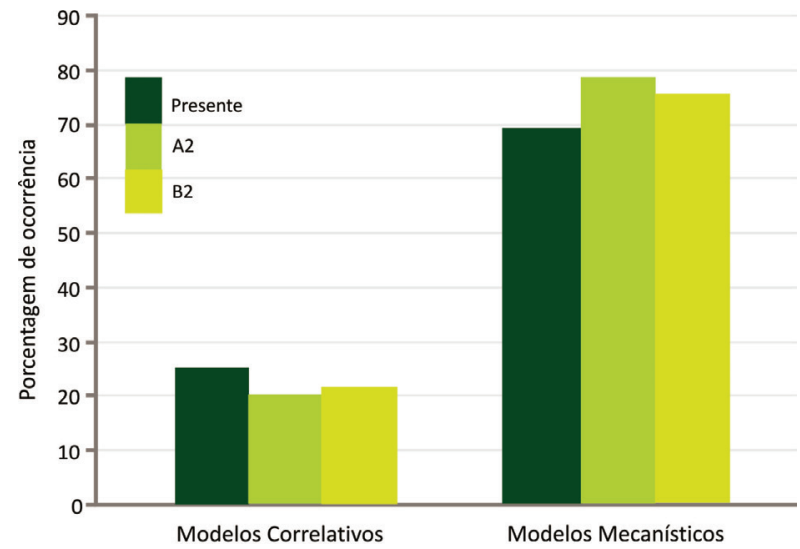

Fig. 4. Porcentagem de ocorrência de Rhinella granulosa (Spix, 1824) estimada pelos modelos de distribuição potencial (correlativos e mecanísticos). de sensitividade, ou seja, proporção de presenças corretamente preditas. A projeção presente apresentou a menor sensitividade $(0,38)$ quando comparada aos cenários pessimista e otimista (A2 $=0,90$ e B2 $=0,87)$. A proporção de ausências preditas corretamente, ou seja, a especificidade, foi relativamente alta e semelhante entre todos os cenários. $\mathrm{O}$ cenário futuro otimista apresentou o melhor desempenho $(\mathrm{B} 2=0,79)$ entre os modelos correlativos.

Em relação aos modelos que consideram a tolerância termal como fator limitante à distribuição geográfica, a sensitividade foi a mais alta possível para todos os cenários, evidenciando que a distribuição potencial de $R$. granulosa foi predita em todos os pontos cuja a espécie foi registrada. No entanto, a especificidade foi baixa para todos os cenários e, consequentemente, o desempenho também se apresentou consideravelmente baixo (entre 0,25 e 0,35 ). Especificamente, o modelo mecanístico que apresentou maior especificidade e desempenho foi o referente ao cenário presente $(0,32$ e 0,35 , respectivamente). Ainda ressalta-se que, entre os cenários futuros, o modelo otimista apresentou maior especificidade e desempenho ( $\mathrm{B} 2=0,25$ e 0,29 , respectivamente).

\section{DISCUSSÃO}

Há grandes evidências de que as mudanças climáticas são uma das maiores ameaças à diversidade de espécies (Araúso et al., 2006; Salazar et al., 2007; Loyola et al., 2008), contudo nossos modelos mostraram que Rhinella granulosa tende a manter sua distribuição sob cenários de mudanças climáticas. Ao contrário de considerar somente cenários presentes (CASSEMIro et al., 2012), nós avaliamos os efeitos das mudanças climáticas, a qual tem sido fator de risco de 
Tab. I. Índices de sensitividade, especificidade e desempenho dos modelos de distribuição potencial (correlativos e mecanísticos) de Rhinella granulosa (Spix, 1824), considerando cenários climáticos presentes e futuros.

\begin{tabular}{lcccc}
\hline Modelos & & Sensitividade & Especificidade & Desempenho \\
\hline \multirow{3}{*}{ Correlativos } & Presente & 0,38 & 0,74 & 0,75 \\
& A2-2080 & 0,90 & 0,76 & 0,77 \\
& B2-2080 & 0,87 & 0,79 & 0,79 \\
\hline \multirow{3}{*}{ Mecanísticos } & Presente & 1 & 0,32 & 0,35 \\
& A2-2080 & 1 & 0,22 & 0,25 \\
& B2-2080 & 1 & 0,25 & 0,29 \\
\hline
\end{tabular}

extinção de muitas espécies de anfíbios (LiPs et al., 2008). Além disso, utilizamos o método de Distância Euclidiana, que nos permite visualizar melhor as áreas de maior adequabilidade ambiental para a espécie e, assim, conseguir prever possíveis deslocamentos de $R$. granulosa frente a um cenário de mudança climática.

No geral os resultados mostraram que os modelos mecanísticos foram menos conservativos do que os correlativos, ou seja, a sua distribuição potencial apresentou-se mais ampla quando fatores fisiológicos foram considerados. Ao considerar tanto os cenários presente quanto os futuros, os modelos de tolerância termal partem do pressuposto que Rhinella granulosa possui alta tolerância fisiológica em relação à temperatura e, consequentemente, tais modelos geraram ampla distribuição potencial. Ao projetarmos a distribuição potencial da espécie em 2080, sob um cenário pessimista, em que as taxas de emissão de carbono e a temperatura são mais elevadas, observamos que a espécie expandiu sua distribuição geográfica. Isso ocorreu porque áreas consideradas frias (p. ex., norte da Argentina e Uruguai) passaram a apresentar temperaturas médias um pouco mais elevadas. De fato, quando o mapa de delta de temperatura é analisado, observamos que essas áreas apresentaram maior diferença de temperatura entre o cenário presente e futuros (2050 e 2080), tornandose áreas potencialmente adequadas à sobrevivência da espécie.

Neste contexto, ARAúJo et al. (2013) afirmam que espécies com maior tolerância ao calor não são estritamente restritas a ambientes mais quentes, além de apresentarem dados de espécies ectotérmicas com grande tolerância ao calor, mas que também suportam ambientes mais frios. Desta forma, não há como descartar que futuramente $R$. granulosa possa vir habitar áreas com temperaturas mínimas baixas, como é mostrado pelos modelos mecanísticos. Contudo, é importante salientar que provavelmente a espécie desenvolverá adaptações fisiológicas e comportamentais para suportar maior tolerância às temperaturas mais baixas. WhitTAKER (1967) afirma que a tolerância das espécies a gradientes ambientais varia continuamente ao longo de tais gradientes e, obviamente, tal tolerância é desenvolvida ao longo de um tempo evolutivo, através da preferência individual (veja também Araúso et al., 2013). Portanto, quando afirmamos que há possibilidade de $R$. granulosa mudar sua tolerância termal no futuro, temos que considerar que além dessa adaptação poder levar milhares de anos, outros fatores (p. ex., bióticos) podem ser cruciais para limitar a sua distribuição.

Contudo, é de se esperar que modelos mecanísticos sobreestimem a distribuição, principalmente onde interações bióticas prevalecem (BUCKLEY et al., 2010; SundaY et al., 2011). Além disso, Narvaes \& Rodrigues (2009) afirmaram que doze espécies pertencentes ao grupo Rhinella $[R$. granulosa, R. pygmaea (Myers \& Carvalho, 1952), R. bergi (Céspedez, 2000), R. major (Muller \& Helmich, 1936), R. mirandaribeiroi (Gallardo, 1965), R. azarai (Gallardo, 1965), R. nattereri (Bokermann, 1967), R. fernandezae (Gallardo, 1957), $R$. dorbignyi (Duméril \& Bibron, 1841), R. merianae (Gallardo, 1965), R. humboldti (Gallardo, 1965) e $R$. centralis Narvaes \& Rodrigues, 2009] apresentam distribuição geográfica que coincide com a distribuição potencial apresentada pelos modelos mecanísticos. Isso pode ser um indicativo de que essas doze espécies estreitamente correlacionadas sejam equivalentes ecológicos, ou seja, possuem requerimentos fisiológicos semelhantes em relação à tolerância termal e até mesmo semelhanças comportamentais e morfológicas. Desta forma, pressupõem-se que $R$. granulosa realmente poderia estar distribuída em toda área estimada pelos três cenários climáticos, porém fatores bióticos (p. ex., interações interespecíficas) e físicos (p. ex., barreiras geográficas) provavelmente estejam delimitando sua ocorrência.

Portanto, é de se esperar que os modelos mecanísticos estimem a distribuição potencial de $R$. granulosa além dos seus registros de ocorrência (veja BUCKLEY et al., 2010 e CASSEMIRO et al., 2012, para mais exemplos). Contudo, anfíbios apresentam baixa habilidade de dispersão e, frequentemente, são inaptos a se locomover para lugares com clima adequado (Munguía et al., 2012); além disso, provavelmente outras variáveis ambientais e interações bióticas influenciem na distribuição realizada da espécie (ANGERT et al., 2011). Diante do exposto, a ampla distribuição apresentada pelos modelos mecanísticos não implica necessariamente em expansão futura da distribuição geográfica, já que outros fatores podem atuar na limitação da dispersão da espécie (mas veja NoRI et al., 2011).

Segundo BeAumont et al. (2005), a adição progressiva de parâmetros climáticos resulta em modelos que geram uma distribuição potencial progressivamente menor, e os resultados mostrados, usando os modelos 
correlativos, corroboram tal afirmação. Acreditamos que o uso de várias variáveis ambientais e suas correlações com os pontos de ocorrência da espécie contribuíram para que as áreas de menor distância do nicho ótimo coincidissem com a área de ocorrência de $R$. granulosa e, assim, conferindo melhor desempenho aos modelos correlativos em relação aos mecanísticos.

Através da abordagem correlativa, observouse que a distribuição potencial nos cenários futuros (principalmente o cenário pessimista) diminuiu, se opondo ao modelo de tolerância termal. Contudo, além da Caatinga, seu habitat natural, a distribuição potencial de $R$. granulosa também foi observada em formas de pequenas manchas no Cerrado. Efeito semelhante foi encontrado por Nori et al. (2011), que observaram redução na adequabilidade de habitat para Rana catesbeiana (Shaw, 1802) em 2080, no entanto, a espécie transferiu sua distribuição para áreas protegidas, onde a adequabilidade ambiental era maior. De acordo com KATZENBERGER et al. (2012), organismos com baixa tolerância ao aquecimento, capacidade de aclimatização limitada e fraca capacidade de dispersão, que lhes permitiriam evitar possíveis condições adversas, sofrerão maior risco de extinção. Além disso, estudos têm demonstrado que espécies tropicais são mais suscetíveis à extinção do que espécies de regiões temperadas quando a tolerância termal é considerada (CALOSI et al., 2008; Deutsch et al., 2008). No entanto, nossos resultados mostraram que $R$. granulosa não terá sua distribuição afetada de forma drástica no futuro; provavelmente isso se deva à tolerância dessa espécie às altas temperaturas, ao contrário do que ocorre com a maioria dos anfíbios, como levantado por outros estudos (CALOSI et al., 2008; Deutsch et al., 2008; KatZenberger et al., 2012). Desta forma, a predição de que espécies de anfíbios distribuídas em climas quentes terão suas distribuições espaciais restringidas por aumento da temperatura não foi observada para $R$. granulosa.

Embora existam pesquisas examinando os efeitos de mudanças da temperatura sobre a dinâmica de populações e comunidades, bem como a evolução fisiológica, tanto em laboratório quanto em mesocosmos (VAN DoORSLAER et al., 2009a,b), ainda não se sabe como sistemas complexos podem responder às mudanças na temperatura (SeArs \& Angilletta, 2011). Em face disso, estudos que integrem aspectos ecológicos e comportamentais aos fisiológicos, levando o histórico de diversificação filogenética e de ocupação dos diferentes biomas, são fundamentais para um melhor entendimento do papel das adaptações da tolerância termal nos padrões atuais de distribuição geográfica e sensibilidade às alterações ambientais (NORI et al., 2011; SEARS \& Angilletta, 2011; KatZennerger et al., 2012; AraúJo \& Peterson, 2012).

Os modelos de nicho são ferramentas computacionais que ainda precisam de certas modificações como a incorporação de fatores bióticos e abióticos; há também uma preocupação na conscientização do uso destes modelos para que não haja falhas de interpretações dos dados. Contudo, os modelos de distribuição apresentados neste estudo contribuem para futuros trabalhos de modelagem, pois esta ferramenta é importante para pesquisas em cenários de mudanças climáticas, contribuindo, assim, para o estudo de espécies mais afetadas por mudanças de temperatura como os anfíbios e, consequentemente, auxiliando na identificação de áreas prioritárias para conservação.

Agradecimentos. Agradecemos a Patrícia Narvaes por gentilmente fornecer os pontos de ocorrência da espécie estudada. Ao CNPq pela bolsa de apoio técnico, concedida à Hauanny Rodrigues Oliveira (Proc. No. 563727/2010-1).

\section{REFERÊNCIAS BIBLIOGRÁFICAS}

Angert, A. L.; Croizier, L. G.; Rissler, L. J.; Gilman, S. E.; Tewksbury, J. J. \& Chunco, A. J. 2011. Do species traits predict recent shifts at expanding range edges? Ecology Letters 14:677-689.

Araújo, M. B. \& Peterson, A. T. 2012. Uses and misuses of bioclimatic envelope modeling. Ecology 93(7):1527-1539.

Araújo, M. B.; Thuiller, W. \& Pearson, R. G. 2006. Climate warming and the decline of amphibians and reptiles in Europe. Journal of Biogeography 33:1712-1728.

Araújo, M. B.; Ferri-Yañez, F.; Bozinovic, F.; Marquet, P. A.; Valladares, F. \& Chown, S. L. 2013. Heat freezes niche evolution. Ecology Letters (in press, doi:10.1111/ele.12155).

Beaumont, L. J.; Hughes, L. \& Pitman, A. 2008. Why is the choice of future climate scenarios for species distribution modelling important? Ecology Letters 11:1135-1146.

Beaumont, L. J.; Hughes, L. \& Poulsen, M. 2005. Predicting species distributions: use of climatic parameters in BIOCLIM and its impact on predictions of species' current and future distributions. Ecological Modelling 186:250-269.

Beaumont, L. J.; Gallagher, R. V.; Thuiller, W.; Downey, P.; Leishman, M. R. \& Hughes L. 2009. Different climatic envelopes among invasive populations may lead to underestimations of current and future biological invasions. Diversity and Distributions 15:409-420.

Brown, J. H.; Stevens, G. C. \& Kaufman, D. M. 1996. The Geographic Range: Size, Shape, Boundaries, and Internal Structure. Annual Review of Ecology and Systematics 27:597-623.

Buckley, L. B.; Urban, M. C.; Angilletta, M. J.; Crozier, L. G.; Rissler, L. J. \& Sears, M. W. 2010. Can mechanism inform species' distribution models? Ecology Letters 13:1041-1054.

Calosi, P.; Bilton, D. T. \& Spicer, J. I. 2008. Thermal tolerance, acclimatory capacity and vulnerability to global climate change. Biology Letters 4:99-102.

CASSEMIRo, F.A. S.; Gouveia, S. F. \& DinIz-Filho, J.A. F. 2012. Distribuição de Rhinella granulosa: integrando envelopes bioclimáticos e respostas ecofisiológicas. Revista da Biologia 8:38-44.

Chen, I. C.; Hill, J. K.; Ohlemuller, R.; Roy, D. B. \& Thomas, C. D. 2011. Rapid Range Shifts of Species Associated with High Levels of Climate Warming. Science 333:1024-1026.

Chown, S. L. \& Gaston, K. J. 2008. Macrophysiology for a changing world. Proceedings of the Royal Society B.275:1469-1478.

Costa, T. R. N.; Carnaval, A. C. O. Q. \& Toledo, L. F. 2012. Mudanças climáticas e seus impactos sobre os anfíbios brasileiros. Revista da Biologia 8:33-37.

De Marco JR, P. \& De Siqueira, M. F. 2009. Como determinar a distribuição de espécies sobre uma abordagem conservacionista. Megadiversidade 5:65-76.

Deutsch, C. A.; Tewksbury, J. J.; Huey, R. B.; Sheldon, K. S.; Ghalambor, C. K.; HaAK, D. C. \& Martin, P. R. 2008. Impacts of climate warming on terrestrial ectotherms across latitude Thermal Safety margin. PNAS 105:6668-6672.

Diniz-Filho, J. A. F.; Bini, L. M.; Rangel, T. F.; Loyola, R. D.; Hof, C.; Nogués-Bravo, D. \& Araújo, M. B. 2009. Partitioning and mapping uncertainties in ensembles of forecasts of species turnover under climate change. Ecography 32:1-10. 
Diniz-Filho, J. A. F; Nabout, J. C.; Bini, L. M.; Loyola, R. D.; Rangel, T. F.; Nogués-Bravo, D. \& AraúJo, M. B. 2010. Ensemble forecasting shifts in climatically suitable areas for Tropidacris cristata (Orthoptera: Acridoidea: Romaleidae). Insect Conservation and Diversity 3:213-221.

Franklin, J. 2010. Mapping Species Distribution. Cambridge, Cambridge University Press. 338p.

Guisan, A. \& Thuiller, W. 2005. Predicting species distribution: offering more than simple habitat models. Ecology Letters 8:9931009.

Hartley, S.; Krushelnycky, P. D. \& Lester, P. J. 2010. Integrating physiology, population dynamics and climate to make multi-scale predictions for the spread of an invasive insect: the Argentine ant at Haleakala National Park, Hawaii. Ecography 33:83-94.

Katzenberger, M.; Tejedo, M.; Duarte, H.; Marangoni, F. \& Beltrán, J. F. 2012. Tolerância e sensibilidade térmica em anfíbios. Revista da Biologia 8:25-32.

Kearney, M. R. \& Porter, W. P. 2009. Mechanistic niche modelling: combining physiological and spatial data to predict species ranges. Ecology Letters 12:334-350.

Kearney, M. R.; Wintle, B. A. \& Porter, W. P. 2010. Correlative and mechanistic models of species distribution provide congruent forecasts under climate change. Conservation Letters 3:203-213.

Kearney.; M. R.; Phillips, B. L.; Tracy, C. R.; Betts, G. \& Porter. W. P. 2008. Modelling species distributions without using species distributions: the cane toad in Australia under current and future climates. Ecography 35:222-234.

Leal, I. R; Tabarelli, M. \& Silva, J. M. C. 2003. Ecologia e conservação da caatinga. Recife, Ed. Universitária da UFPE. $822 \mathrm{p}$

Lips, K. R.; Diffendorfer, J.; Mendelson, J. R. III \& SeArs, M. W. 2008. Riding the Wave: Reconciling the Roles of Disease and Climate Change in Amphibian Declines. PLoS Biology 6:e72

Loyola, R. D. \& Martins, R. P. 2011. Small-scale area effect on species richness and nesting occupancy of cavity-nesting bees and wasps. Revista Brasileira de Entomologia 55:69-74.

Loyola, R. D.; Becker, C. G.; Kubota, U.; Haddad, C. F. B. \& LEWINSOHN, T. M. 2008. Hung out to dry, choice of priority ecoregions for conserving threatened Neotropical anurans depends on life-history traits. PLoS ONE 3:e2120.

Manel, S.; Williams, H. C. \& Ormerod, S. J. 2001. Evaluating presence - absence models in ecology: the need to account for prevelance. Journal of Applied Ecology 38:921-931.

Munguía, M.; Rahbek, C.; Rangel, T. F.; Diniz-Filho, J. A. F. \& Araúso, M. B. 2012. Equilibrium of Global Amphibian Species Distributions with Climate. PLoS ONE 17:1-9.

NarvaEs, P. \& Rodrigues, M. T. 2009. Taxonomic revision of Rhinella granulosa species group (Amphibia, Anura, Bufonidae), with a description of a new species. Arquivos de Zoologia 40:1-73.

Navas, C.; Gomes, F. \& Carvalho, J. 2008. Thermal relationships and exercise physiology in anuran amphibians: Integration and evolutionary implications? Comparative Biochemistry and Physiology. A, Molecular \& Integrative Physiology 151:344362.

Navas, C. A.; Antoniazzi, M. M.; Carvalho, J. E.; Suzuki, H. \& JARED, C. 2007. Physiological basis for diurnal activity in dispersing juvenile Bufo granulosus in the Caatinga, a Brazilian semi-arid environment. Comparative Biochemistry and Physiology. Part A, Molecular \& Integrative Physiology 147:647-657.
Nori, J.; Urbina-Cardona, J. N.; Loyola, R. D.; Lescano, J. N. \& LeYnaud, G. C. 2011. Climate Change and American Bullfrog Invasion: What Could We Expect in South America? PLoS ONE 6:e25718.

Parmesan, C. 2006. Ecological and evolutionary responses to recent climate change. Annual Review of Ecology and Systematics 37:637-669.

Rangel, T. F. L. V. B.; Diniz-Filho, J. A. F.; \& AraúJo, M. B. 2009. BIOENSEMBLES 1.0. Software for Computer Intensive Ensemble Forecasting of Species Distributions Under Climate Change. Goiás, Madrid, Évora

RibeIRO, P. L. \& NAVAS, C. 2012. A macrofisiologia e sua importância em estudos em mudanças climáticas. Revista da Biologia 8:1-4.

Rodrigues, M. T. 2003. Herpetofauna da Caatinga. In: LeAL, I. R. TABARELli, M. \& Silva, J. M. C. eds. Ecologia e Conservação da Caatinga. Recife, Universidade Federal de Pernambuco. p.181236.

Root, T. L.; Price, J. T.; Hall, K. R.; Schneider, S. H.; Rosenzweig, C. \& Pounds, J. A. 2003. Finger prints of global warming on wild animals and plants. Nature 421:57-60.

Salazar, L. F.; Nobre, C. A. \& Oyama, M. D. 2007. Climate change consequences on the distribution in tropical South America. Geophysical Research Letters 34:1-6.

Sears, M. W. \& Angilletta, M. J. 2011. Responses of organisms to climate change: a synthetic approach to the role of thermal adaptation. Integrative and Comparative Biology 51:662-665

Sinervo, B.; Méndez-de-la Cruz, F.; Miles, D. B.; Heulin, B. Bastiaans, E.; Villagrán- Santa Cruz, M.; Resendiz, R. L.; Mendez, N. M.; Calderón- Espinosa, M. L.; Meza- Lázaro, R. N.; Gadsden, H.; Avila, L. J.; Morando, M.; Riva, I. J.; Sepulveda, P. V.; Rocha, C. F. D.; Ibarguengoytía, N.; Puntriano, C. A.; Massot, M.; Lepetez, V.; Oksanen, T. A Chapple, D. G.; Bauer, A. M.; Branch, W. R.; Clobert, J. \& SITES JR, J. W. 2010. Erosion of Lizard Diversity by Climate Change and Altered Thermal Niches. Science 5980:894-899.

Soberón, J. 2007. Grinnellian and Eltonian niches and geographic distributions of species. Ecology Letters 10:1115-1123.

Sunday, J. M.; Crim, R. N.; Harley, C. D. G. \& Hart, M. W. 2011 Quantifying Rates of Evolutionary Adaptation in Response to Ocean Acidification. PLoS ONE 6:1-8.

Thomas, C. D. 2010. Climate, climate change and range boundaries. Diversity and Distributions 16:488-495.

TRENBERTH, K. E. 2005. Uncertainty in hurricanes and global warming. Science 308:1753-1754

Van Doorslaer, W.; Vanoverbeke, J.; Duvivier, C. \& Rousseaux, S. 2009a. Local adaptation to higher temperatures reduces immigration success of genotypes from a warmer region in the water flea Daphnia. Global Change Biology 15:3046-3055.

Van Doorslaer, W.; Stoks, R.; Duvivier, C.; Bednarska, A. \& De Meester, L. 2009b. Population dynamics determine genetic adaptation to temperature in Daphnia. Evolution 63:1867-1878.

Walther, G. R.; Post, E.; Convey, P; Menzel, A; Parmesan, C.; Beebee, T. J. C.; Fromentin, J. M.; Guldberg, O. H. \& BAIRLEIN, F. 2002. Ecological responses to recent climate change. Nature 416:389-395.

WhitTAKer, R.; Willis, K. J. \& Field, R. 2001. Scale and species richness: towards a general, hierarchical theory of species diversity. Journal of Biogeography 28:453-470.

WhitTAKer, R. H. 1967. Gradient analysis of vegetation. Biological Reviews 49:207-264

Recebido em 21 de abril de 2013. Aceito em 6 de agosto de 2013. ISSN 0073-4721

Artigo disponível em: www.scielo.br/isz 